This postprint is published in:

Applicable analysis, Volume 96, Number 9, 2017, 1483-1496

DOI: $10.1080 / 00036811.2016 .1221069$

\title{
MULTIPLE SOLUTIONS OF NONLINEAR EQUATIONS INVOLVING THE SQUARE ROOT OF THE LAPLACIAN
}

\author{
GIOVANNI MOLICA BISCI, DUŠAN REPOVŠ, AND LUCA VILASI
}

\begin{abstract}
In this paper we examine the existence of multiple solutions of parametric fractional equations involving the square root of the Laplacian $A_{1 / 2}$ in a smooth bounded domain $\Omega \subset \mathbb{R}^{n}(n \geq 2)$ and with Dirichlet zero-boundary conditions, i.e.

$$
\begin{cases}A_{1 / 2} u=\lambda f(u) & \text { in } \Omega \\ u=0 & \text { on } \partial \Omega .\end{cases}
$$

The existence of at least three $L^{\infty}$-bounded weak solutions is established for certain values of the parameter $\lambda$ requiring that the nonlinear term $f$ is continuous and with a suitable growth. Our approach is based on variational arguments and a variant of Caffarelli-Silvestre's extension method.
\end{abstract}

\section{INTRODUCTION}

In this paper we study under the variational viewpoint the existence of multiple (weak) solutions of the following nonlocal problem

$$
\begin{cases}A_{1 / 2} u=\lambda \beta(x) f(u) & \text { in } \Omega \\ u=0 & \text { on } \partial \Omega\end{cases}
$$

where $\Omega$ is a bounded open subset of $\mathbb{R}^{n}(n \geq 2)$ with Lipschitz boundary $\partial \Omega, \lambda$ is a positive real parameter and $\beta: \Omega \rightarrow \mathbb{R}$ is a function belonging to $L^{\infty}(\Omega)$ and satisfying

$$
\beta_{0}:=\underset{x \in \Omega}{\operatorname{essinf}} \beta(x)>0 .
$$

The fractional operator $A_{1 / 2}$ that appears in $\left(P_{\lambda}\right)$ is defined by using the approach developed in the pioneering works of Caffarelli \& Silvestre [4], Caffarelli \& Vasseur [5], Cabré \& Tan [3], to which we refer in Section 2 for the precise mathematical description and related properties. As it was pointed out in [3], the fractions of the Laplacian, like the square root of the Laplacian $A_{1 / 2}$, are the infinitesimal generators of Lévy stable diffusion processes and they appear, among the other things, in anomalous diffusions in plasmas, flames propagation, population dynamics, geophysical fluid dynamics and American options in finance.

\footnotetext{
Key words and phrases. Fractional Laplacian, variational methods, multiple solutions. 2010 AMS Subject Classification: Primary: 49J35, 35A15, 35S15; Secondary: 47G20, $45 \mathrm{G} 05$.
} 
Elliptic equations involving fractional powers of the Laplacian have recently been treated in $[1,2,7,8,10,16]$ (see also the references therein). In particular, Cabré \& Tan in [3] have studied the existence, non-existence and regularity of positive solutions of problem $\left(P_{\lambda}\right)$ with power-type nonlinearities, together with à priori estimates of Gidas-Spruck type and symmetry results of Gidas-Ni-Nirenberg type. Along the same direction we mention the paper [17] by Tan.

In the current context, regarding the nonlinear term, we assume that $f: \mathbb{R} \rightarrow \mathbb{R}$ is continuous and

there exist two non-negative constants $a_{1}, a_{2}$ and $q \in(1,2 n /(n-1))$ such that

$$
|f(t)| \leq a_{1}+a_{2}|t|^{q-1}
$$

for every $t \in \mathbb{R}$;

the potential $F(t):=\int_{0}^{t} f(\xi) d \xi$ satisfies the sign-condition

$$
\inf _{t \in[0,+\infty)} F(t) \geq 0
$$

in addition to some technical, yet central algebraic assumptions that will be stated later on (see (31)).

A special case of our main result, which ensures the multiplicity of positive solutions when $\lambda$ is big enough, reads as follows:

Theorem 1.1. Let $r>0$ and denote

$$
\Gamma_{r}^{0}:=\left\{(x, 0) \in \partial \mathbb{R}_{+}^{n+1}:|x|<r\right\},
$$

where $\partial \mathbb{R}_{+}^{n+1}:=\mathbb{R}^{n} \times(0,+\infty)$ and $n \geq 2$. Let $f:[0,+\infty) \rightarrow \mathbb{R}$ be a continuous function such that

$$
f(t) \leq a_{2} t^{m}
$$

for all $t \in[0,+\infty)$ and for some $m \in(1,(n+1) /(n-1))$. Furthermore, assume that there is $\zeta>0$ such that $f(t)>0$ for every $t \in(0, \zeta)$ and $f(\zeta)=0$.

Then there exists $\lambda^{\star}>0$ such that, for every $\lambda>\lambda^{\star}$, the following nonlocal problem

$$
\begin{cases}A_{1 / 2} u=\lambda \beta(x) f(u) & \text { in } \Gamma_{r}^{0} \\ u>0 & \text { on } \Gamma_{r}^{0} \\ u=0 & \text { on } \partial \Gamma_{r}^{0},\end{cases}
$$

admits at least two distinct weak solutions $u_{1, \lambda}, u_{2, \lambda} \in L^{\infty}\left(\Gamma_{r}^{0}\right) \cap H_{0}^{1 / 2}\left(\Gamma_{r}^{0}\right)$.

The above theorem is the fractional analogue, on the $n$-dimensional Euclidean ball, of a classical multiplicity result due to Ambrosetti \& Rabinowitz (see [13, Theorem 2.32]).

In the more general Theorem 3.4 we determine a precise (bounded) interval of positive parameters $\lambda$ for which the nonlocal problem $\left(P_{\lambda}\right)$ admits 
at least three $L^{\infty}$-bounded weak solutions in a suitable fractional Sobolev space $X_{0}^{1 / 2}(\Omega)$ (see Section 2). Such a result is proved by exporting certain variational techniques to the fractional framework. Indeed, following the paper [3], we first transform problem $\left(P_{\lambda}\right)$ to a local problem in a cylinder $\mathcal{C}_{\Omega}$, by using the notion of harmonic extension and the Dirichlet-to-Neumann map on $\Omega$ (cf. Section 2). Successively, we study the existence of critical points of the energy functional $\mathcal{J}_{\lambda}$ associated with the extended problem. A local minimum result for differentiable functionals ([14]), the classical minimization technique and a critical point result due to Pucci and Serrin ([12, Theorem 4]) are the abstract tools behind the existence of such critical points.

Indeed, the first weak solution turns out to be a local minimum $w_{1, \lambda}$ for the energy functional $\mathcal{J}_{\lambda}$ (Proposition 3.1); the second one, say $w_{2, \lambda}$, is obtained as a global minimum of $\mathcal{J}_{\lambda}$ as a byproduct of the sequential weak lower semicontinuity and coercivity of $\mathcal{J}_{\lambda}$. Finally, thanks to Propositions 3.2 and 3.3 , we are able to distinguish them, that is $w_{1, \lambda} \neq w_{2, \lambda}$, and since $\mathcal{J}_{\lambda}$ fulfills the Palais-Smale condition, Pucci-Serrin's result guarantees the existence of a third critical point $w_{3, \lambda} \notin\left\{w_{1, \lambda}, w_{2, \lambda}\right\}$ (see Theorem 3.4).

The traces of these solutions give back three weak solutions to $\left(P_{\lambda}\right)$ that are bounded in $L^{\infty}(\Omega)$ owing to [3, Theorem 5.2].

The paper is structured as follows. In Section 2 we introduce notation, preliminary notions and variational framework. In Section 3 we state and prove our main result with some applications.

\section{Preliminaries}

We start by recalling the functional space setting naturally associated with $\left(P_{\lambda}\right)$, first introduced in [3]; we also refer to the recent book [9], as well as [6], for detailed accounts on Sobolev spaces of fractional order.

2.1. Fractional Sobolev spaces. The power $A_{1 / 2}$ of the Laplace operator $-\Delta$ in a bounded domain $\Omega$ with zero-boundary conditions is defined via the spectral decomposition, using the powers of the eigenvalues of the original operator. Hence, according to classical results on positive operators in $\Omega$,

if $\left\{\left(\varphi_{j}, \lambda_{j}\right)\right\}_{j \in \mathbb{N}}$ are the eigenfunctions and eigenvalues of the usual linear Dirichlet problem

$$
\begin{cases}-\Delta u=\lambda u & \text { in } \Omega \\ u=0 & \text { on } \partial \Omega\end{cases}
$$

then $\left\{\left(\varphi_{j}, \lambda_{j}^{1 / 2}\right)\right\}_{j \in \mathbb{N}}$ are the eigenfunctions and eigenvalues of the corresponding fractional one:

$$
\begin{cases}A_{1 / 2} u=\lambda u & \text { in } \Omega \\ u=0 & \text { on } \partial \Omega\end{cases}
$$


As usual we consider each eigenvalue $\lambda_{j}$ repeated according to its (finite) multiplicity,

$$
0<\lambda_{1}<\lambda_{2} \leq \cdots \leq \lambda_{j} \leq \lambda_{j+1} \leq \cdots
$$

It is well known that $\lambda_{j} \rightarrow+\infty$ as $j \rightarrow+\infty$. Moreover, we can suppose the eigenfunctions $\left\{\varphi_{j}\right\}_{j \in \mathbb{N}}$ are normalized as follows:

$$
\int_{\Omega}\left|\nabla \varphi_{j}(x)\right|^{2} d x=\lambda_{j} \int_{\Omega}\left|\varphi_{j}(x)\right|^{2} d x=\lambda_{j}, \quad \forall j \in \mathbb{N},
$$

and

$$
\int_{\Omega} \nabla \varphi_{i}(x) \cdot \nabla \varphi_{j}(x) d x=\int_{\Omega} \varphi_{i}(x) \varphi_{j}(x) d x=0, \quad \forall i \neq j .
$$

Finally, standard regularity arguments ensure that $\varphi_{j} \in C^{2}(\bar{\Omega})$ for every $j \in \mathbb{N}$.

The operator $A_{1 / 2}$ turns out to be well-defined on the Sobolev space

$$
H_{0}^{1 / 2}(\Omega):=\left\{u \in L^{2}(\Omega): u=\sum_{j=1}^{\infty} a_{j} \varphi_{j} \text { and } \sum_{j=1}^{\infty} a_{j}^{2} \lambda_{j}^{1 / 2}<+\infty\right\},
$$

endowed with the norm

$$
\|u\|_{H_{0}^{1 / 2}(\Omega)}:=\left(\sum_{j=1}^{\infty} a_{j}^{2} \lambda_{j}^{1 / 2}\right)^{1 / 2}
$$

and it has the following form

$$
A_{1 / 2} u:=\sum_{j=1}^{\infty} a_{j} \lambda_{j}^{1 / 2} \varphi_{j}, \quad \forall u \in H_{0}^{1 / 2}(\Omega) .
$$

2.2. The extension problem. Associated with the bounded domain $\Omega$, let us consider the cylinder

$$
\mathcal{C}_{\Omega}:=\{(x, y): x \in \Omega, y>0\} \subset \mathbb{R}_{+}^{n+1},
$$

and denote by $\partial_{L} \mathcal{C}_{\Omega}:=\partial \Omega \times[0,+\infty)$ its lateral boundary.

For a function $u \in H_{0}^{1 / 2}(\Omega)$ define the harmonic extension $\mathrm{E}(u)$ to $\mathcal{C}_{\Omega}$ as the solution of the problem

$$
\begin{cases}\operatorname{div}(\nabla \mathrm{E}(u))=0 & \text { in } \mathcal{C}_{\Omega} \\ \mathrm{E}(u)=0 & \text { on } \partial_{L} \mathcal{C}_{\Omega} \\ \operatorname{Tr}(\mathrm{E}(u))=u & \text { on } \Omega\end{cases}
$$

where

$$
\operatorname{Tr}(\mathrm{E}(u))(x):=\mathrm{E}(u)(x, 0), \quad \forall x \in \Omega .
$$

The extension function $\mathrm{E}(u)$ belongs to the Hilbert space $X_{0}^{1 / 2}\left(\mathcal{C}_{\Omega}\right):=\left\{w \in L^{2}\left(\mathcal{C}_{\Omega}\right): w=0\right.$ on $\left.\partial_{L} \mathcal{C}_{\Omega}, \int_{\mathcal{C}_{\Omega}}|\nabla w(x, y)|^{2} d x d y<+\infty\right\}$, 
equipped with the standard norm

$$
\|w\|_{X_{0}^{1 / 2}\left(\mathcal{C}_{\Omega}\right)}:=\left(\int_{\mathcal{C}_{\Omega}}|\nabla w(x, y)|^{2} d x d y\right)^{1 / 2}
$$

which can also be characterized as

$$
X_{0}^{1 / 2}\left(\mathcal{C}_{\Omega}\right)=\left\{w \in L^{2}\left(\mathcal{C}_{\Omega}\right): w=\sum_{j=1}^{\infty} b_{j} \varphi_{j} e^{-\lambda_{j}^{1 / 2} y} \text { with } \sum_{j=1}^{\infty} b_{j}^{2} \lambda_{j}^{1 / 2}<+\infty\right\},
$$

see [3, Lemma 2.10].

In our framework, a crucial relationship between the spaces $X_{0}^{1 / 2}\left(\mathcal{C}_{\Omega}\right)$ and $H_{0}^{1 / 2}(\Omega)$ introduced above is played by the trace operator $\operatorname{Tr}: X_{0}^{1 / 2}\left(\mathcal{C}_{\Omega}\right) \rightarrow$ $H_{0}^{1 / 2}(\Omega)$ defined by

$$
\operatorname{Tr}(w)(x):=w(x, 0), \quad \forall x \in \Omega .
$$

which turns out to be a continuous map (see [3, Lemma 2.6]). We also notice that

$$
H_{0}^{1 / 2}(\Omega)=\left\{u \in L^{2}(\Omega): u=\operatorname{Tr}(w) \text { for some } w \in X_{0}^{1 / 2}\left(\mathcal{C}_{\Omega}\right)\right\} \subset H^{1 / 2}(\Omega),
$$

and that the extension operator $\mathrm{E}: H_{0}^{1 / 2}(\Omega) \rightarrow X_{0}^{1 / 2}\left(\mathcal{C}_{\Omega}\right)$ is an isometry, i.e.

$$
\|\mathrm{E}(u)\|_{X_{0}^{1 / 2}\left(\mathcal{C}_{\Omega}\right)}=\|u\|_{H_{0}^{1 / 2}(\Omega)},
$$

for every $u \in H_{0}^{1 / 2}(\Omega)$. Here $H^{1 / 2}(\Omega)$ denotes the Sobolev space of order $1 / 2$ defined by

$$
H^{1 / 2}(\Omega):=\left\{u \in L^{2}(\Omega): \int_{\Omega \times \Omega} \frac{|u(x)-u(y)|^{2}}{|x-y|^{n+1}} d x d y<+\infty\right\},
$$

with the norm

$$
\|u\|_{H^{1 / 2}(\Omega)}:=\left(\int_{\Omega \times \Omega} \frac{|u(x)-u(y)|^{2}}{|x-y|^{n+1}} d x d y+\int_{\Omega}|u(x)|^{2} d x\right)^{1 / 2} .
$$

Clearly, for every $w \in X_{0}^{1 / 2}\left(\mathcal{C}_{\Omega}\right)$ the following trace inequality holds

$$
\|\operatorname{Tr}(w)\|_{H_{0}^{1 / 2}(\Omega)} \leq\|w\|_{X_{0}^{1 / 2}\left(\mathcal{C}_{\Omega}\right)}
$$

while, due to [3, Lemmas 2.4 and 2.5], the embedding $\operatorname{Tr}\left(X_{0}^{1 / 2}\left(\mathcal{C}_{\Omega}\right)\right) \hookrightarrow$ $L^{p}(\Omega)$ is continuous for any $p \in\left[1,2^{\sharp}\right]$ and compact whenever $p \in\left[1,2^{\sharp}\right)$, where $2^{\sharp}:=2 n /(n-1)$ denotes the fractional critical Sobolev exponent. Thus, if $p \in\left[1,2^{\sharp}\right]$, then there exists a positive constant $c_{p}$ (depending on $p$, $n$ and $|\Omega|)$ such that

$$
\left(\int_{\Omega}|\operatorname{Tr}(w)(x)|^{p} d x\right)^{1 / p} \leq c_{p}\left(\int_{\mathcal{C}_{\Omega}}|\nabla w(x, y)|^{2} d x d y\right)^{1 / 2},
$$

for every $w \in X_{0}^{1 / 2}\left(\mathcal{C}_{\Omega}\right)$. 
As we have briefly outlined in the introduction, we will adopt the following equivalent definition of the square root of the Laplacian (see for instance $[1,2,3])$. By using the extension $\mathrm{E}(u) \in X_{0}^{1 / 2}\left(\mathcal{C}_{\Omega}\right)$ of a function $u \in H_{0}^{1 / 2}(\Omega)$, i.e. the solution of (7), the fractional operator $A_{1 / 2}$ in $\Omega$ acting on $u$ agrees with the map

$$
A_{1 / 2} u(x):=-\lim _{y \rightarrow 0^{+}} \frac{\partial \mathrm{E}(u)(x, y)}{\partial y} \quad \forall x \in \Omega,
$$

i.e.

$$
A_{1 / 2} u(x)=\frac{\partial \mathrm{E}(u)(x, 0)}{\partial \nu} \quad \forall x \in \Omega
$$

where $\nu$ is the unit outer normal to $\mathcal{C}_{\Omega}$ at $\Omega \times\{0\}$.

2.3. Weak solutions. Fix $\lambda>0$ and assume that $f: \mathbb{R} \rightarrow \mathbb{R}$ is continuous and satisfies (2). We say that a function $u=\operatorname{Tr}(w) \in H_{0}^{1 / 2}(\Omega)$ is a weak solution of $\left(P_{\lambda}\right)$ if $w \in X_{0}^{1 / 2}\left(\mathcal{C}_{\Omega}\right)$ weakly solves

$$
\begin{cases}-\operatorname{div}(\nabla w)=0 & \text { in } \mathcal{C}_{\Omega} \\ w=0 & \text { on } \partial_{L} \mathcal{C}_{\Omega} \\ \frac{\partial w}{\partial \nu}=\lambda \beta(x) f(\operatorname{Tr}(w)) & \text { on } \Omega,\end{cases}
$$

i.e.

$$
\int_{\mathcal{C}_{\Omega}}\langle\nabla w, \nabla \varphi\rangle d x d y=\lambda \int_{\Omega} \beta(x) f(\operatorname{Tr}(w)(x)) \operatorname{Tr}(\varphi)(x) d x,
$$

for every $\varphi \in X_{0}^{1 / 2}\left(\mathcal{C}_{\Omega}\right)$.

As direct computations prove, equation (11) represents the variational formulation of (10) and the energy functional $\mathcal{J}_{\lambda}: X_{0}^{1 / 2}\left(\mathcal{C}_{\Omega}\right) \rightarrow \mathbb{R}$ associated with (11) is defined by

$$
\mathcal{J}_{\lambda}(w):=\frac{1}{2} \int_{\mathcal{C}_{\Omega}}|\nabla w(x, y)|^{2} d x d y-\lambda \int_{\Omega} \beta(x) F(\operatorname{Tr}(w)(x)) d x,
$$

for every $w \in X_{0}^{1 / 2}\left(\mathcal{C}_{\Omega}\right)$. Indeed, under our assumptions on the nonlinear term, it is straightforward to show that $\mathcal{J}_{\lambda}$ is well-defined and of class $C^{1}$ in $X_{0}^{1 / 2}\left(\mathcal{C}_{\Omega}\right)$ and that its critical points are exactly the weak solutions of problem (10). The traces of such critical points being weak solutions of $\left(P_{\lambda}\right)$, we can employ methods from critical point theory to attack problem $\left(P_{\lambda}\right)$.

To this end, the following abstract theorem due to Ricceri (see [14]), restated here in a more convenient form, plays a key role in our study.

Theorem 2.1. Let $X$ be a reflexive real Banach space, and let $\Phi, \Psi: X \rightarrow \mathbb{R}$ be two Gâteaux differentiable functionals such that $\Phi$ is strongly continuous, sequentially weakly lower semicontinuous and coercive. Furthermore, assume that $\Psi$ is sequentially weakly upper semicontinuous. For every $r>$ 
$\inf _{X} \Phi$, put

$$
\varphi(r):=\inf _{w \in \Phi^{-1}((-\infty, r))} \frac{\left(\sup _{z \in \Phi^{-1}((-\infty, r))} \Psi(z)\right)-\Psi(w)}{r-\Phi(w)} .
$$

Then for each $r>\inf _{X} \Phi$ and each $\lambda \in(0,1 / \varphi(r))$, the restriction of $J_{\lambda}:=$ $\Phi-\lambda \Psi$ to $\Phi^{-1}((-\infty, r))$ admits a global minimum, which is a critical point (local minimum) of $J_{\lambda}$ in $X$.

In addition to the above result, the following classical theorem by Pucci and Serrin (see [12, Theorem 4] and [13, Theorem 3.10]) will allow us to deduce the existence of a further critical point.

Theorem 2.2. Let $J: X \rightarrow \mathbb{R}$ be a $C^{1}$-functional satisfying the (PS) condition. If $J$ has a pair of local minima or maxima, then $J$ admits a third critical point.

For the sake of completeness we recall that, if $X$ is a real Banach space, a $C^{1}$-functional $J: X \rightarrow \mathbb{R}$ is said to satisfy the Palais-Smale condition at level $\mu \in \mathbb{R}$ when

$(\mathrm{PS})_{\mu}$ Every sequence $\left\{z_{j}\right\}_{j \in \mathbb{N}} \subset X$ such that

$$
J\left(z_{j}\right) \rightarrow \mu \quad \text { and } \quad\left\|J^{\prime}\left(z_{j}\right)\right\|_{X^{*}} \rightarrow 0
$$

as $j \rightarrow+\infty$, possesses a convergent subsequence in $X$.

Here $X^{*}$ denotes the topological dual of $X$. We say that $J$ satisfies the Palais-Smale condition ((PS) for short) if (PS) $)_{\mu}$ holds for every $\mu \in \mathbb{R}$.

\section{The MAIN RESUlt}

Define the functionals $\Phi, \Psi: X_{0}^{1 / 2}\left(\mathcal{C}_{\Omega}\right) \rightarrow \mathbb{R}$, naturally associated with $\left(P_{\lambda}\right)$, by

$$
\Phi(w):=\frac{1}{2}\|w\|_{X_{0}^{1 / 2}\left(\mathcal{C}_{\Omega}\right)}^{2}, \quad \Psi(w):=\int_{\Omega} \beta(x) F(\operatorname{Tr}(w)(x)) d x, \quad \forall w \in X_{0}^{1 / 2}\left(\mathcal{C}_{\Omega}\right) .
$$

Clearly, $\Phi$ is a coercive, continuously Gâteaux-differentiable and sequentially weakly lower semicontinuous functional. On the other hand, $\Psi$ is welldefined, continuously Gâteaux-differentiable and, on account of (2) and the compactness of the embedding

$$
\operatorname{Tr}\left(X_{0}^{1 / 2}\left(\mathcal{C}_{\Omega}\right)\right) \hookrightarrow L^{p}(\Omega), \quad p \in\left[1,2^{\sharp}\right),
$$

also weakly continuous in $X_{0}^{1 / 2}\left(\mathcal{C}_{\Omega}\right)$.

It is easy to deduce that

$\Phi^{\prime}(w)(\varphi)=\int_{\mathcal{C}_{\Omega}}\langle\nabla w, \nabla \varphi\rangle d x d y$ and $\Psi^{\prime}(w)(\varphi)=\int_{\Omega} \beta(x) f(\operatorname{Tr}(w)(x)) \operatorname{Tr}(\varphi)(x) d x$,

for every $\varphi \in X_{0}^{1 / 2}\left(\mathcal{C}_{\Omega}\right)$. 
The aim of this section is to establish a precise interval of values of the parameter $\lambda$ for which the functional $\mathcal{J}_{\lambda}$ admits at least three critical points.

To this end, fix a point $x_{0} \in \Omega$ and choose $\tau>0$ so that

$$
B\left(x_{0}, \tau\right):=\left\{x \in \mathbb{R}^{n}:\left|x-x_{0}\right|<\tau\right\} \subseteq \Omega .
$$

Define also

$$
g_{\Omega}^{(n)}:=\frac{2^{n}-1}{2^{n-1}} \tau^{n-2} \omega_{n}, \quad h_{\Omega}^{(n)}:=g_{\Omega}^{(n)}+\frac{|\Omega|}{8}
$$

and

$$
K_{1}:=\frac{\sqrt{2} c_{1} h_{\Omega}^{(n)}\|\beta\|_{\infty}}{\omega_{n} \beta_{0}}\left(\frac{2}{\tau}\right)^{n}, \quad K_{2}:=\frac{2^{q / 2} c_{q}^{q} h_{\Omega}^{(n)}\|\beta\|_{\infty}}{q \omega_{n} \beta_{0}}\left(\frac{2}{\tau}\right)^{n},
$$

where

$$
\omega_{n}:=\frac{\pi^{n / 2}}{\Gamma\left(1+\frac{n}{2}\right)}
$$

denotes the Lebesgue measure of the unit ball in $\mathbb{R}^{n}$ and

$$
\Gamma(t):=\int_{0}^{+\infty} z^{t-1} e^{-z} d z, \quad \forall t>0,
$$

is the classical gamma function.

Theorem 3.1. Let $f: \mathbb{R} \rightarrow \mathbb{R}$ be a function satisfying (2). Then for every $\gamma>0$ and every $\lambda<\mu_{2}$, with

$$
\mu_{2}:=\frac{2^{n}}{\tau^{n} \omega_{n} \beta_{0}}\left(\frac{h_{\Omega}^{(n)} \gamma}{a_{1} K_{1}+a_{2} K_{2} \gamma^{q-1}}\right),
$$

there exists a local minimum $w_{1, \lambda} \in \Phi^{-1}\left(\left(-\infty, \gamma^{2}\right)\right)$ of $\mathcal{J}_{\lambda}$ in $X_{0}^{1 / 2}\left(\mathcal{C}_{\Omega}\right)$.

Proof. Owing to the growth condition (2), one has

$$
F(t) \leq a_{1}|t|+\frac{a_{2}}{q}|t|^{q},
$$

for every $t \in \mathbb{R}$. With the idea of using Theorem 2.1, let us consider the function

$$
\chi(r):=\frac{\sup _{z \in \Phi^{-1}((-\infty, r])} \Psi(z)}{r},
$$

with $r \in(0,+\infty)$. It follows from (16) and (9) that, for each $z \in X_{0}^{1 / 2}\left(\mathcal{C}_{\Omega}\right)$,

$$
\begin{aligned}
\Psi(z) & =\int_{\Omega} \beta(x) F(\operatorname{Tr}(z)(x)) d x \\
& \leq\left(a_{1}\|\operatorname{Tr}(z)\|_{L^{1}(\Omega)}+\frac{a_{2}}{q}\|\operatorname{Tr}(z)\|_{L^{q}(\Omega)}^{q}\right)\|\beta\|_{\infty} \\
& \leq\left(a_{1} c_{1}\|z\|_{X_{0}^{1 / 2}\left(\mathcal{C}_{\Omega}\right)}+\frac{a_{2}}{q} c_{q}^{q}\|z\|_{X_{0}^{1 / 2}\left(\mathcal{C}_{\Omega}\right)}^{q}\right)\|\beta\|_{\infty}
\end{aligned}
$$


and therefore

$$
\sup _{z \in \Phi^{-1}((-\infty, r])} \Psi(z) \leq \sqrt{2 r} a_{1} c_{1}\|\beta\|_{\infty}+\frac{(2 r)^{q / 2} a_{2} c_{q}^{q}}{q}\|\beta\|_{\infty} .
$$

The above inequality yields

$$
\chi(r) \leq \sqrt{\frac{2}{r}} a_{1} c_{1}\|\beta\|_{\infty}+\frac{2^{q / 2} a_{2} c_{q}^{q}}{q} r^{q / 2-1}\|\beta\|_{\infty}
$$

for every $r>0$ and therefore

$$
\begin{aligned}
\chi\left(\gamma^{2}\right)=\frac{\sup _{z \in \Phi^{-1}\left(\left(-\infty, \gamma^{2}\right]\right)} \Psi(z)}{\gamma^{2}} & \leq \sqrt{2} \frac{a_{1} c_{1}}{\gamma}\|\beta\|_{\infty}+\frac{2^{q / 2} a_{2} c_{q}^{q}}{q} \gamma^{q-2}\|\beta\|_{\infty} \\
& =\beta_{0} \frac{\tau^{n}}{2^{n}} \frac{\omega_{n}}{h_{\Omega}^{(n)}}\left(a_{1} \frac{K_{1}}{\gamma}+a_{2} K_{2} \gamma^{q-2}\right) \\
& =\frac{1}{\mu_{2}} .
\end{aligned}
$$

Now, bearing in mind that $0 \in \Phi^{-1}\left(\left(-\infty, \gamma^{2}\right)\right)$ and $\Phi(0)=\Psi(0)=0$, we observe that

$$
\varphi\left(\gamma^{2}\right):=\inf _{w \in \Phi^{-1}\left(\left(-\infty, \gamma^{2}\right)\right)} \frac{\left(\sup _{z \in \Phi^{-1}\left(\left(-\infty, \gamma^{2}\right)\right)} \Psi(z)\right)-\Psi(w)}{\gamma^{2}-\Phi(w)} \leq \chi\left(\gamma^{2}\right)
$$

and thus

$$
\lambda \in\left(0, \mu_{2}\right) \subseteq\left(0,1 / \varphi\left(\gamma^{2}\right)\right) .
$$

Then, in the light of Theorem 2.1 there exists a function $w_{1, \lambda} \in \Phi^{-1}\left(\left(-\infty, \gamma^{2}\right)\right)$ such that

$$
\mathcal{J}_{\lambda}^{\prime}\left(w_{1, \lambda}\right)=\Phi^{\prime}\left(w_{1, \lambda}\right)-\lambda \Psi^{\prime}\left(w_{1, \lambda}\right)=0
$$

and, in particular, $w_{1, \lambda}$ is a global minimum of the restriction of $\mathcal{J}_{\lambda}$ to $\Phi^{-1}\left(\left(-\infty, \gamma^{2}\right)\right)$. This completes the proof.

For the sequel we need to define suitable test functions in the space $X_{0}^{1 / 2}\left(\mathcal{C}_{\Omega}\right)$. Take two positive constants $\gamma$ and $\varrho$ such that

$$
\varrho>\frac{\gamma}{\sqrt{g_{\Omega}^{(n)}}}
$$

and define the truncated cones $\omega_{\tau}^{\varrho}: \Omega \rightarrow \mathbb{R}$ as follows:

$$
\omega_{\tau}^{\varrho}(x):= \begin{cases}0 & \text { if } x \in \bar{\Omega} \backslash B\left(x_{0}, \tau\right) \\ \frac{2 \varrho}{\tau}\left(\tau-\left|x-x_{0}\right|\right) & \text { if } x \in B\left(x_{0}, \tau\right) \backslash B\left(x_{0}, \tau / 2\right) \\ \varrho & \text { if } x \in B\left(x_{0}, \tau / 2\right) .\end{cases}
$$


It is easily seen that

$$
\begin{aligned}
\int_{\Omega}\left|\nabla \omega_{\tau}^{\varrho}(x)\right|^{2} d x & =\int_{B\left(x_{0}, \tau\right) \backslash B\left(x_{0}, \tau / 2\right)} \frac{4 \varrho^{2}}{\tau^{2}} d x \\
& =\frac{4 \varrho^{2}}{\tau^{2}}\left(\left|B\left(x_{0}, \tau\right)\right|-\left|B\left(x_{0}, \tau / 2\right)\right|\right) \\
& =4 \varrho^{2} \omega_{n} \tau^{n-2}\left(1-\frac{1}{2^{n}}\right) .
\end{aligned}
$$

Let

$$
w_{\tau}^{\varrho}(x, y):=e^{-\frac{y}{2}} \omega_{\tau}^{\varrho}(x), \quad \forall(x, y) \in \mathcal{C}_{\Omega} .
$$

Clearly, $w_{\tau}^{\varrho} \in X_{0}^{1 / 2}\left(\mathcal{C}_{\Omega}\right)$ and, since

$$
\left|\nabla w_{\tau}^{\varrho}(x, y)\right|^{2}=e^{-y}\left|\nabla \omega_{\tau}^{\varrho}(x)\right|^{2}+\frac{1}{4} e^{-y}\left|\omega_{\tau}^{\varrho}(x)\right|^{2}, \quad \forall(x, y) \in \mathcal{C}_{\Omega}
$$

it follows that

$$
\begin{aligned}
\left\|w_{\tau}^{\varrho}\right\|_{X_{0}^{1 / 2}\left(\mathcal{C}_{\Omega}\right)}^{2} & :=\int_{\mathcal{C}_{\Omega}}\left|\nabla w_{\tau}^{\varrho}(x, y)\right|^{2} d x d y \\
& =\int_{\mathcal{C}_{\Omega}} e^{-y}\left|\nabla \omega_{\tau}^{\varrho}(x)\right|^{2} d x d y+\frac{1}{4} \int_{\mathcal{C}_{\Omega}} e^{-y}\left|\omega_{\tau}^{\varrho}(x)\right|^{2} d x d y \\
& =\int_{0}^{+\infty} e^{-y} d y\left(\int_{\Omega}\left|\nabla \omega_{\tau}^{\varrho}(x)\right|^{2} d x+\frac{1}{4} \int_{\Omega}\left|\omega_{\tau}^{\varrho}(x)\right|^{2} d x\right) \\
& =\int_{\Omega}\left|\nabla \omega_{\tau}^{\varrho}(x)\right|^{2} d x+\frac{1}{4} \int_{\Omega}\left|\omega_{\tau}^{\varrho}(x)\right|^{2} d x
\end{aligned}
$$

Thus, (20) and (21) provide the estimate

$$
4 \omega_{n} \tau^{n-2}\left(1-\frac{1}{2^{n}}\right) \varrho^{2} \leq\left\|w_{\tau}^{\varrho}\right\|_{X_{0}^{1 / 2}\left(\mathcal{C}_{\Omega}\right)}^{2} \leq\left(4 \omega_{n} \tau^{n-2}\left(1-\frac{1}{2^{n}}\right)+\frac{|\Omega|}{4}\right) \varrho^{2} .
$$

Define

$$
\mu_{1}:=\left(\frac{2^{n} h_{\Omega}^{(n)}}{\omega_{n} \tau^{n} \beta_{0}}\right) \frac{\varrho^{2}}{F(\varrho)} .
$$

Proposition 3.2. The following inequality holds

$$
\Phi\left(w_{\tau}^{\varrho}\right)>\gamma^{2} .
$$

In addition, assuming that $\mu_{1}<\mu_{2}$ and $\lambda \in\left(\mu_{1}, \mu_{2}\right)$, one has

$$
\Phi\left(w_{\tau}^{\varrho}\right)-\lambda \Psi\left(w_{\tau}^{\varrho}\right)<\gamma^{2}-\lambda \sup _{w \in \Phi^{-1}\left(\left(-\infty, \gamma^{2}\right]\right)} \Psi(w) .
$$

Proof. The estimate (23) follows at once from (19) and (22).

From (3) we infer

$$
\int_{\Omega} \beta(x) F\left(\operatorname{Tr}\left(w_{\tau}^{\varrho}\right)(x)\right) d x \geq \beta_{0} \omega_{n} \frac{\tau^{n}}{2^{n}} F(\varrho),
$$


which, together with (22), gives

$$
\frac{\Psi\left(w_{\tau}^{\varrho}\right)}{\Phi\left(w_{\tau}^{\varrho}\right)} \geq \beta_{0} \frac{\omega_{n}}{h_{\Omega}^{(n)}} \frac{\tau^{n}}{2^{n}} \frac{F(\varrho)}{\varrho^{2}} .
$$

Since $\mu_{1}<\lambda<\mu_{2}$, we obtain

$$
\chi\left(\gamma^{2}\right) \leq \frac{1}{\mu_{2}}<\frac{1}{\lambda}<\frac{1}{\mu_{1}} \leq \frac{\Psi\left(w_{\tau}^{\varrho}\right)}{\Phi\left(w_{\tau}^{\varrho}\right)}
$$

and therefore

$$
\begin{aligned}
\frac{\Psi\left(w_{\tau}^{\varrho}\right)-\sup _{w \in \Phi^{-1}\left(\left(-\infty, \gamma^{2}\right]\right)} \Psi(w)}{\Phi\left(w_{\tau}^{\varrho}\right)-\gamma^{2}} & \geq \frac{\Psi\left(w_{\tau}^{\varrho}\right)-\gamma^{2} \frac{\Psi\left(w_{\tau}^{\varrho}\right)}{\Phi\left(w_{\tau}^{\varrho}\right)}}{\Phi\left(w_{\tau}^{\varrho}\right)-\gamma^{2}} \\
& =\frac{\Psi\left(w_{\tau}^{\varrho}\right)}{\Phi\left(w_{\tau}^{\varrho}\right)} \\
& \geq \frac{1}{\mu_{1}}>\frac{1}{\lambda} .
\end{aligned}
$$

This implies inequality (24) and the proof is thus completed.

Associated with $\gamma$ let us consider, for every $w \in X_{0}^{1 / 2}\left(\mathcal{C}_{\Omega}\right)$, the following truncated functional

$$
\mathcal{J}_{\lambda}^{(\gamma)}(w):= \begin{cases}\gamma^{2}-\lambda \Psi(w) & \text { if } w \in \Phi^{-1}\left(\left(-\infty, \gamma^{2}\right]\right) \\ \mathcal{J}_{\lambda}(w) & \text { if } w \notin \Phi^{-1}\left(\left(-\infty, \gamma^{2}\right]\right)\end{cases}
$$

Fixing $\lambda>0$, since $\mathcal{J}_{\lambda}^{(\gamma)}$ is sequentially weakly lower semicontinuous and coercive on the Hilbert space $X_{0}^{1 / 2}\left(\mathcal{C}_{\Omega}\right)$, it will attain a global minimum $w_{2, \lambda}$, that is:

$$
\mathcal{J}_{\lambda}^{(\gamma)}\left(w_{2, \lambda}\right) \leq \mathcal{J}_{\lambda}^{(\gamma)}(w), \quad \forall w \in X_{0}^{1 / 2}\left(\mathcal{C}_{\Omega}\right)
$$

The next result states the impossibility for $w_{2, \lambda}$ to lie in the ball $B(0, \sqrt{2} \gamma)$ when $\lambda \in\left(\mu_{1}, \mu_{2}\right)$.

Theorem 3.3. Assume $\lambda \in\left(\mu_{1}, \mu_{2}\right)$. Then

$$
w_{2, \lambda} \notin \Phi^{-1}\left(\left(-\infty, \gamma^{2}\right]\right)
$$

and $\mathcal{J}_{\lambda}^{\prime}\left(w_{2, \lambda}\right)=0$, i.e. $w_{2, \lambda} \in X_{0}^{1 / 2}\left(\mathcal{C}_{\Omega}\right)$ is a critical point of $\mathcal{J}_{\lambda}$.

Proof. Pick $\lambda \in\left(\mu_{1}, \mu_{2}\right)$ and, arguing by contradiction, assume that

$$
w_{2, \lambda} \in \Phi^{-1}\left(\left(-\infty, \gamma^{2}\right]\right) \text {. }
$$

In view of $(28)$ it follows that

$$
\gamma^{2}-\lambda \Psi\left(w_{2, \lambda}\right) \leq \mathcal{J}_{\lambda}\left(w_{\tau}^{\varrho}\right)
$$

while due to Proposition 3.2 one has

$$
\mathcal{J}_{\lambda}\left(w_{\tau}^{\varrho}\right)<\gamma^{2}-\lambda \Psi\left(w_{2, \lambda}\right)
$$

Collecting inequalities (29) and (30) we get the desired contradiction. 
We are now in position to prove our main result.

Theorem 3.4. Let $\Omega$ be an open bounded set of $\mathbb{R}^{n}(n \geq 2)$ with Lipschitz boundary $\partial \Omega, \beta: \Omega \rightarrow \mathbb{R}$ an $L^{\infty}$-map satisfying (1) and $f: \mathbb{R} \rightarrow \mathbb{R} a$ continuous function satifying (2) and (3). Furthermore, assume that the following algebraic inequality holds

$$
\frac{F(\varrho)}{\varrho^{2}}>a_{1} \frac{K_{1}}{\gamma}+a_{2} K_{2} \gamma^{q-2}
$$

for some $\varrho, \gamma>0$ satifying (19), in addition to

$$
F(t) \leq b\left(1+|t|^{l}\right)
$$

for all $t \in \mathbb{R}$ and for some positive constants $b$ and $l<2$.

Then for each $\lambda \in\left(\mu_{1}, \mu_{2}\right)$, problem $\left(P_{\lambda}\right)$ has at least three weak solutions $u_{1, \lambda}, u_{2, \lambda}, u_{3, \lambda} \in L^{\infty}(\Omega) \cap H_{0}^{1 / 2}(\Omega)$.

Proof. It is easy to verify that condition (31) forces $\mu_{1}<\mu_{2}$. Then fixing $\lambda \in\left(\mu_{1}, \mu_{2}\right)$ and appealing to Theorems 3.1 and 3.3, we can deduce the existence of the first two solutions, recalling that $u_{j, \lambda}=\operatorname{Tr}\left(w_{j, \lambda}\right), j=1,2$. The nature of local minima of the functions $w_{1, \lambda}, w_{2, \lambda}$ permits us to apply Theorem 2.2. Let us show that $\mathcal{J}_{\lambda}$ satisfies $(\mathrm{PS})_{\mu}$ for $\mu \in \mathbb{R}$. Let $\left\{w_{j}\right\}_{j \in \mathbb{N}} \subset$ $X_{0}^{1 / 2}\left(\mathcal{C}_{\Omega}\right)$ satisfy

$$
\mathcal{J}_{\lambda}\left(w_{j}\right) \rightarrow \mu \quad \text { and } \quad\left\|\mathcal{J}_{\lambda}^{\prime}\left(w_{j}\right)\right\|_{*} \rightarrow 0
$$

as $j \rightarrow+\infty$, where

$\left\|\mathcal{J}_{\lambda}^{\prime}\left(w_{j}\right)\right\|_{*}:=\sup \left\{\left|\left\langle\mathcal{J}_{\lambda}^{\prime}\left(w_{j}\right), \varphi\right\rangle\right|: \varphi \in X_{0}^{1 / 2}\left(\mathcal{C}_{\Omega}\right) \quad\right.$ and $\left.\quad\|\varphi\|_{X_{0}^{1 / 2}\left(\mathcal{C}_{\Omega}\right)}=1\right\}$.

Since $l<2$, for every $w \in X_{0}^{1 / 2}\left(\mathcal{C}_{\Omega}\right)$ one has $|\operatorname{Tr}(w)|^{l} \in L^{2 / l}(\Omega)$ and combined with Hölder's inequality this gives

$$
\int_{\Omega}|\operatorname{Tr}(w)(x)|^{l} d x \leq|\Omega|^{\frac{2-l}{2}}\|\operatorname{Tr}(w)\|_{L^{2}(\Omega)}^{l} \quad \forall w \in X_{0}^{1 / 2}\left(\mathcal{C}_{\Omega}\right)
$$

and, by (9),

$$
\int_{\Omega}|\operatorname{Tr}(w)(x)|^{l} d x \leq c_{2}^{l}|\Omega|^{\frac{2-l}{2}}\|w\|_{X_{0}^{1 / 2}\left(\mathcal{C}_{\Omega}\right)}^{l} \quad \forall w \in X_{0}^{1 / 2}\left(\mathcal{C}_{\Omega}\right) .
$$

In the light of inequalities (32) and (33) we get

$\mathcal{J}_{\lambda}(w) \geq \frac{1}{2}\|w\|_{X_{0}^{1 / 2}\left(\mathcal{C}_{\Omega}\right)}^{2}-\lambda b c_{2}^{l}\|\beta\|_{\infty}|\Omega|^{\frac{2-l}{2}}\|w\|_{X_{0}^{1 / 2}\left(\mathcal{C}_{\Omega}\right)}^{l}-\lambda b\|\beta\|_{\infty}|\Omega| \quad \forall w \in X_{0}^{1 / 2}\left(\mathcal{C}_{\Omega}\right)$,

and therefore $\mathcal{J}_{\lambda}$ is bounded from below and $\mathcal{J}_{\lambda}(w) \rightarrow+\infty$ as $\|w\|_{X_{0}^{1 / 2}\left(\mathcal{C}_{\Omega}\right)} \rightarrow$ $+\infty$. This allows us to deduce that the sequence $\left\{w_{j}\right\}_{j \in \mathbb{N}}$ is bounded in $X_{0}^{1 / 2}\left(\mathcal{C}_{\Omega}\right)$. Since $X_{0}^{1 / 2}\left(\mathcal{C}_{\Omega}\right)$ is reflexive we can extract a subsequence, for simplicity denoted again $\left\{w_{j}\right\}_{j \in \mathbb{N}}$, such that $w_{j} \rightarrow w_{\infty}$ in $X_{0}^{1 / 2}\left(\mathcal{C}_{\Omega}\right)$, i.e.,

$$
\int_{\mathcal{C}_{\Omega}}\left\langle\nabla w_{j}, \nabla \varphi\right\rangle d x d y \rightarrow \int_{\mathcal{C}_{\Omega}}\left\langle\nabla w_{\infty}, \nabla \varphi\right\rangle d x d y
$$


as $j \rightarrow+\infty$ for any $\varphi \in X_{0}^{1 / 2}\left(\mathcal{C}_{\Omega}\right)$.

We will prove that $w_{j} \rightarrow w_{\infty}$ as $j \rightarrow+\infty$. Keeping (13) in mind, one has (35)

$\left\langle\Phi^{\prime}\left(w_{j}\right), w_{j}-w_{\infty}\right\rangle=\left\langle\mathcal{J}_{\lambda}^{\prime}\left(w_{j}\right), w_{j}-w_{\infty}\right\rangle+\lambda \int_{\Omega} \beta(x) f\left(\operatorname{Tr}\left(w_{j}\right)(x)\right) \operatorname{Tr}\left(w_{j}-w_{\infty}\right)(x) d x$,

where

$$
\left\langle\Phi^{\prime}\left(w_{j}\right), w_{j}-w_{\infty}\right\rangle=\int_{\mathcal{C}_{\Omega}}\left|\nabla w_{j}(x, y)\right|^{2} d x d y-\int_{\mathcal{C}_{\Omega}}\left\langle\nabla w_{j}, \nabla w_{\infty}\right\rangle d x d y .
$$

Since $\left\|\mathcal{J}_{\lambda}^{\prime}\left(w_{j}\right)\right\|_{*} \rightarrow 0$ and the sequence $\left\{w_{j}-w_{\infty}\right\}_{j \in \mathbb{N}}$ is bounded in $X_{0}^{1 / 2}\left(\mathcal{C}_{\Omega}\right)$, taking into account the fact that $\left|\left\langle\mathcal{J}_{\lambda}^{\prime}\left(w_{j}\right), w_{j}-w_{\infty}\right\rangle\right| \leq\left\|\mathcal{J}_{\lambda}^{\prime}\left(w_{j}\right)\right\|_{*} \| w_{j}-$ $w_{\infty} \|_{X_{0}^{1 / 2}\left(\mathcal{C}_{\Omega}\right)}$, one has

$$
\left\langle\mathcal{J}_{\lambda}^{\prime}\left(w_{j}\right), w_{j}-w_{\infty}\right\rangle \rightarrow 0
$$

as $j \rightarrow+\infty$. Furthermore, by (2) and Hölder's inequality one has

$$
\begin{aligned}
\int_{\Omega} \beta(x)\left|f\left(\operatorname{Tr}\left(w_{j}\right)(x)\right)\right|\left|\operatorname{Tr}\left(w_{j}-w_{\infty}\right)(x)\right| d x & \leq a_{1}\|\beta\|_{\infty} \int_{\Omega}\left|\operatorname{Tr}\left(w_{j}-w_{\infty}\right)(x)\right| d x \\
& +a_{2}\|\beta\|_{\infty} \int_{\Omega}\left|\operatorname{Tr}\left(w_{j}\right)(x)\right|^{q-1}\left|\operatorname{Tr}\left(w_{j}-w_{\infty}\right)(x)\right| d x \\
& \leq a_{1}\|\beta\|_{\infty}\left\|\operatorname{Tr}\left(w_{j}-w_{\infty}\right)\right\|_{1} \\
& +a_{2}\|\beta\|_{\infty}\left\|\operatorname{Tr}\left(w_{j}\right)\right\|_{q}^{q-1}\left\|\operatorname{Tr}\left(w_{j}-w_{\infty}\right)\right\|_{q} .
\end{aligned}
$$

Since the embeddings $\operatorname{Tr}\left(X_{0}^{1 / 2}\left(\mathcal{C}_{\Omega}\right)\right) \hookrightarrow L^{1}(\Omega), \operatorname{Tr}\left(X_{0}^{1 / 2}\left(\mathcal{C}_{\Omega}\right)\right) \hookrightarrow L^{q}(\Omega)$ are compact, we obtain

$$
\int_{\Omega} \beta(x)\left|f\left(\operatorname{Tr}\left(w_{j}\right)(x)\right)\right|\left|\operatorname{Tr}\left(w_{j}-w_{\infty}\right)(x)\right| d x \rightarrow 0
$$

as $j \rightarrow+\infty$.

Relations (36) and (37) force

$$
\left\langle\Phi^{\prime}\left(w_{j}\right), w_{j}-w_{\infty}\right\rangle \rightarrow 0
$$

as $j \rightarrow+\infty$ and hence

$$
\int_{\mathcal{C}_{\Omega}}\left|\nabla w_{j}(x, y)\right|^{2} d x d y-\int_{\mathcal{C}_{\Omega}}\left\langle\nabla w_{j}, \nabla w_{\infty}\right\rangle d x d y \rightarrow 0
$$

as $j \rightarrow+\infty$. Thus it follows by (34) and (39) that

$$
\lim _{j \rightarrow+\infty} \int_{\mathcal{C}_{\Omega}}\left|\nabla w_{j}(x, y)\right|^{2} d x d y=\int_{\mathcal{C}_{\Omega}}\left|\nabla w_{\infty}(x, y)\right|^{2} d x d y,
$$

as desired, and the third solution to $\left(P_{\lambda}\right)$ is obtained as well. Finally, due to (2) and the essential boundedness of $\beta$, by [3, Theorem 5.2] we get $u_{i, \lambda}:=$ $\operatorname{Tr}\left(w_{i, \lambda}\right) \in L^{\infty}(\Omega)$ for $i \in\{1,2,3\}$. 
As a corollary of the previous result, we can deduce Theorem 1.1 stated in the introduction.

Proof of Theorem 1.1. Let us consider the non-negative continuous function $f^{\star}: \mathbb{R} \rightarrow \mathbb{R}$ defined by

$$
f^{\star}(t):= \begin{cases}f(t) & \text { if } 0<t \leq \zeta \\ 0 & \text { otherwise }\end{cases}
$$

and apply Theorem 3.4 to the problem

$$
\begin{cases}A_{1 / 2} u=\lambda \beta(x) f^{\star}(u) & \text { in } \Gamma_{r}^{0} \\ u>0 & \text { on } \Gamma_{r}^{0} \\ u=0 & \text { on } \partial \Gamma_{r}^{0} .\end{cases}
$$

Now, fixing

$$
\lambda>\frac{2^{n} h_{\Omega}^{(n)}}{\omega_{n} \tau^{n} \beta_{0}} \inf _{0<\varrho \leq \zeta} \frac{\varrho^{2}}{F(\varrho)}
$$

there exists $\bar{\varrho}>0$ such that

$$
\lambda>\frac{2^{n} h_{\Omega}^{(n)}}{\omega_{n} \tau^{n} \beta_{0}} \frac{\bar{\varrho}^{2}}{F(\bar{\varrho})} .
$$

If we take

$$
\gamma<\min \left\{\sqrt{g_{\Omega}^{(n)}} \bar{\varrho},\left(\frac{2^{n} h_{\Omega}^{(n)}}{a_{2} \omega_{n} \tau^{n} \beta_{0} K_{2} \lambda}\right)^{1 /(m-1)}\right\},
$$

it is easily seen that all the assumptions of Theorem 3.4 are satisfied. Hence there exist at least two weak solutions of problem (40) and, in turn, of problem (4).

Remark 3.5. We point out that the operator $A_{1 / 2}$ we considered here is not to be confused with the integro-differential operator defined, up to a constant, by

$$
(-\Delta)^{1 / 2} u(x):=-\int_{\mathbb{R}^{n}} \frac{u(x+y)+u(x-y)-2 u(x)}{|y|^{n+1}} d y, \quad \forall x \in \mathbb{R}^{n} .
$$

In fact, Servadei \& Valdinoci in [15] showed that these two operators, though often denoted the same way, are really different, with eigenvalues and eigenfunctions behaving quite differently (see also Musina \& Nazarov [11]).

Remark 3.6. The techniques adopted in this paper are still valid if we consider the more general problem:

$$
\left(P_{\alpha, \lambda}\right) \quad \begin{cases}A_{\alpha / 2} u=\lambda \beta(x) f(u) & \text { in } \Omega \\ u=0 & \text { on } \partial \Omega\end{cases}
$$


with $\alpha \in(0,2)$. The operator $A_{\alpha / 2}$ is defined by

$$
A_{\alpha / 2} u(x):=-\kappa_{\alpha} \lim _{y \rightarrow 0^{+}} y^{1-\alpha} \frac{\partial \mathrm{E}(u)(x, y)}{\partial y} \quad \forall x \in \Omega,
$$

where $u$ belongs to the space

$H_{0}^{\alpha / 2}(\Omega):=\left\{u \in L^{2}(\Omega): u=\sum_{j=1}^{\infty} a_{j} \varphi_{j}\right.$ and $\left.\|u\|_{H_{0}^{\alpha / 2}(\Omega)}:=\left(\sum_{j=1}^{\infty} a_{j}^{2} \lambda_{j}^{\alpha / 2}\right)^{1 / 2}<+\infty\right\}$,

and its $\alpha$-harmonic extension $\mathrm{E}_{\alpha}(u)$ to the cylinder $C_{\Omega}$ is the unique solution of the local problem

$$
\begin{cases}\operatorname{div}\left(y^{1-\alpha} \nabla \mathrm{E}_{\alpha}(u)\right)=0 & \text { in } \mathcal{C}_{\Omega} \\ \mathrm{E}_{\alpha}(u)=0 & \text { on } \partial_{L} \mathcal{C}_{\Omega} \\ \operatorname{Tr}\left(\mathrm{E}_{\alpha}(u)\right)=u & \text { on } \Omega\end{cases}
$$

Such an extension lies in the space

$$
X_{0}^{\alpha / 2}\left(\mathcal{C}_{\Omega}\right):=\left\{w \in L^{2}\left(\mathcal{C}_{\Omega}\right): w=0 \text { on } \partial_{L} \mathcal{C}_{\Omega},\|w\|_{X_{0}^{\alpha / 2}\left(\mathcal{C}_{\Omega}\right)}<+\infty\right\},
$$

where

$$
\|w\|_{X_{0}^{\alpha / 2}\left(\mathcal{C}_{\Omega}\right)}:=\left(\kappa_{\alpha} \int_{\mathcal{C}_{\Omega}} y^{1-\alpha}|\nabla w(x, y)|^{2} d x d y\right)^{1 / 2}
$$

and

$$
\kappa_{\alpha}:=\frac{\Gamma\left(\frac{\alpha}{2}\right)}{2^{1-\alpha} \Gamma\left(1-\frac{\alpha}{2}\right)}
$$

is a normalization constant which makes the operator $\mathrm{E}_{\alpha}: H_{0}^{1 / 2}(\Omega) \rightarrow$ $X_{0}^{1 / 2}\left(\mathcal{C}_{\Omega}\right)$ an isometry. As a class of test functions in $X_{0}^{\alpha / 2}\left(\mathcal{C}_{\Omega}\right)$ necessary for our approach one can choose (recall that $\omega_{\tau}^{\varrho}$ is defined by $(3)$ )

$$
w_{\tau}^{\varrho}(x, y):=\frac{1-e^{-\alpha y}}{\alpha y} \omega_{\tau}^{\varrho}(x), \quad \forall(x, y) \in \mathcal{C}_{\Omega} .
$$

\section{ACKnowledgements}

The paper has been carried out under the auspices of the 2016 INdAMGNAMPA project Problemi variazionali su varietà Riemanniane e gruppi di Carnot and the Slovenian Research Agency grants P1-0292, J1-7025, J16721, and J1-5435.

\section{REFERENCES}

[1] B. Barrios, E. Colorado, A. De Pablo, and U. Sánchez, On some critical problems for the fractional Laplacian operator, J. Differential Equations, 252, no. 11, (2012), 6133-6162.

[2] C. Brändle, E. Colorado, A. de Pablo, and U. Sánchez, A concave-convex elliptic problem involving the fractional Laplacian, Proc. Roy. Soc. Edinburgh Sect. A, 143, no. 1 (2013), 39-71. 
[3] X. Cabré And J. TAn, Positive solutions of nonlinear problems involving the square root of the Laplacian, Adv. Math., 224, no. 5 (2010), 2052-2093.

[4] L. Caffarelli And L. Silvestre, An extension problem related to the fractional Laplacian, Comm. Partial Differential Equations 32, no. 7-9 (2007), 1245-1260.

[5] L. Caffarelli and A. Vasseur, Drift diffusion equation with fractional diffusion and the quasi-geostrophic equation, Ann. of Math. 171, no. 2 (2010), 903-930.

[6] E. Di Nezza, G. Palatucci And E. VAldinoci, Hitchhiker's guide to the fractional Sobolev spaces, Bull. Sci. Math. 136, no. 5 (2012), 521-573.

[7] M. Marinelli And D. Mugnai, The generalized logistic equation with indefinite weight driven by the square root of the Laplacian, Nonlinearity 27 (2014), no. 9, 2361-2376.

[8] G. Molica Bisci And V. Rădulescu, A sharp eigenvalue theorem for fractional elliptic equations, Israel J. Math. (2017). DOI: 10.1007/s11856-017-1482-2

[9] G. Molica Bisci, V. Rădulescu, and R. Servadei, Variational Methods for Nonlocal Fractional Problems. With a Foreword by Jean Mawhin, Encyclopedia of Mathematics and its Applications, Cambridge University Press, 162 Cambridge, 2016.

[10] D. Mugnai And D. Pagliardini, Existence and multiplicity results for the fractional Laplacian in bounded domains, Adv. Calc. Var. (2017). DOI: 10.1515/acv-2015-0032

[11] R. Musina And Nazarov, On fractional Laplacians, Commun. Partial Differential Equations 39 (2014), 1780-1790.

[12] P. Pucci And J. Serrin, Extensions of the mountain pass theorem, J. Funct. Anal. 59 (1984), no. 2, 185-210.

[13] P.H. Rabinowitz, Minimax methods in critical point theory with applications to differential equations, CBMS Reg. Conf. Ser. Math., 65, American Mathematical Society, Providence, RI (1986).

[14] B. RICCERI, A general variational principle and some of its applications, J. Comput. Appl. Math. 113 (2000), 401-410.

[15] R. Servadei And E. VAldinOci, On the spectrum of two different fractional operators, Proc. Roy. Soc. Edinburgh Sect. A 144 (2014), 1-25.

[16] J. TAN, The Brezis-Nirenberg type problem involving the square root of the Laplacian, Calc. Var. Partial Differential Equations 36, no. 1-2 (2011), 21-41.

[17] J. TAn, Positive solutions for non local elliptic problems, Discrete Contin. Dyn. Syst. 33, no. 2 (2013), 837-859.

(G. Molica Bisci) Dipartimento P.A.U., Università Degli Studi Mediterranea di Reggio Calabria, Salita Melissari - Feo di Vito, 89100 Reggio Calabria, ITALY

E-mail address: gmolica@unirc.it

(D. Repovš) Faculty of Education and Faculty of Mathematics and Physics, University of Ljubljana \& Institute of Mathematics, Physics and Mechanics, 1000 Ljubluana, Slovenia

E-mail address: dusan.repovs@guest.arnes.si

(L. Vilasi) Department of Mathematical and Computer Sciences, Physical Sciences and Earth Sciences, University of Messina, Viale F. Stagno d'Alcontres 31, 98166 Messina, ItAly

E-mail address: lvilasi@unime.it 